\title{
DIFFERENTIATION IN THE UPPER MANTLE DEDUCED FROM MINERALS FOUND IN THE KIMBERLITE OF THE ROBERTS VICTOR MINE IN THE BOSHOF-DEALESVILLE AREA
}

\section{G.J. Geringer, Department of Geology, U.O.F.S.}

The kimberlite body of the Roberts Victor Mine is situated some $40 \mathrm{~km}$ from Boshof in the western Orange Free State. It consists of two parallel fissures, No. 1 and No. 2 .

The kimberlite has a porphyritic texture and contains xenocrysts of olivine, garnet, pyroxene and phlogopite in a fine grained groundmass of phlogopite, calcite and magnetite. Inclusion-rich and inclusion-poor kimberlite occur in this locality. A great variety of inclusions of which some ultramafic xenoliths are probably derived from the upper parts of the upper mantle, are present.

The ultramafic xenoliths in the kimberlite of the Rovic Mine can be classified as garnetite, eclogite (Group A), phlogopite-eclogite, garnet-pyroxenite and garnet-peridotite.

A comparative study, carried out on 67 garnet xenocrysts and on garnets recovered from ultramafic xenoliths indicates that the xenocrysts represent monomineralic fragments of crushed xenoliths. Investigation of phlogopite xenocrysts, phlogopite from ultramafic xenoliths and phlogopite from the groundmass indicates that phlogopite xenocrysts are also derived from ultramafic xenoliths. Pyroxene is a major constituent of the ultramafic xenoliths, some of which contain chrome diopside. Chrome diopside is also found in the tailings of the concentration plant. The pyroxene xenocrysts are therefore also remnants of crushed xenoliths.

According to the composition of the garnet, phlogopite and pyroxene as well as the presence of diamond in ultramafic xenoliths it is postulated that they are derived from a deep-seated source probably from the upper mantle.

The position of the various layers in the upper mantle can be deduced from the optical properties of garnet, phlogopite and pyroxene. At the top almandine-pyrope, with a refractive index of $1,7670-1,7730$ occurs. This zone is followed by a zone containing pyrope with refractive index of $1,7560-1,7620$. Below this zone, chrome-pyrope 
with refractive index of $1,7410-1,7560$ is present.

The pyroxene present consists of chrome-diopside, diopside and omphacite, In the upper parts of the upper mantle omphacite with refractive index $\beta=1,6896$ is present. This omphacite is rich in the diopside component ( $64 \%$ diopside, $36 \%$ jadeite). In the deeper parts of the upper mantle omphacite with refractive index $\beta=1,6790$ occurs. This pyroxene consists of $28 \%$ diopside, $68 \%$ jadeite and $4 \%$ acmite.

The refractive indices of phlogopite decreases with increasing depth in the upper mantle. This can be due to an increasing titanium and decreasing iron content in the phlogopite.

Detailed work also showed that the specific gravity of the ultramafic xenoliths increases with increasing depth.

A differentiated upper mantle is proposed consisting of garnetperidotite at the top, followed by garnet-pyroxenite and then by eclogite of Group A. Below this zone phlogopite-eclogite and diamondiferous eclogite might occur (Table I). 


\begin{tabular}{|c|c|c|c|c|c|c|c|c|c|}
\hline \multicolumn{2}{|c|}{ 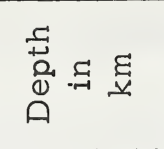 } & 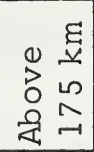 & 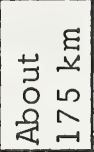 & 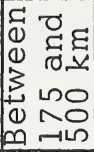 & 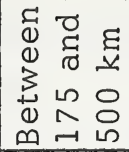 & 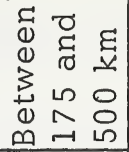 & $\begin{array}{cc}3 & E \\
3 & \underline{y} \\
0 & 0 \\
0 & 0 \\
0 & 0\end{array}$ & $\begin{array}{l}3 \\
3 \\
0 \\
0 \\
0 \\
0 \\
0 \\
0\end{array}$ & $\begin{array}{l}3 \frac{E}{y} \\
0 \\
0 \\
0 \\
0 \\
0\end{array}$ \\
\hline ச் & 足 & 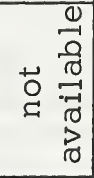 & 1 & $\begin{array}{c}\infty \\
\sim \\
\sim\end{array}$ & $\begin{array}{l}\circ \\
\dot{+} \\
\dot{m}\end{array}$ & $\begin{array}{l}\infty \\
+ \\
\dot{m}\end{array}$ & n' & $\begin{array}{l}\text { ठ'। } \\
\text { N। }\end{array}$ & 1 \\
\hline \multirow{2}{*}{ 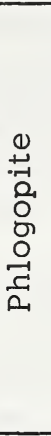 } & $\begin{array}{l}E \\
0 \\
-ت \\
\pm=1 \\
02 \\
0 \\
0 \\
E \\
0 \\
0 \\
0\end{array}$ & 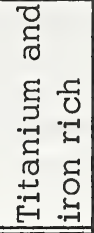 & 1 & 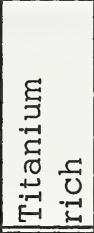 & 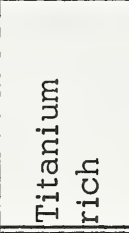 & I & 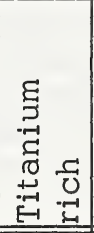 & 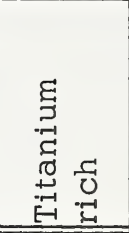 & 1 \\
\hline & 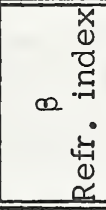 & $\begin{array}{l}\text { N } \\
0 \\
0 \\
-i\end{array}$ & 1 & $\begin{array}{l}\text { ㅇ } \\
\text { के } \\
\text { ம } \\
\text { - }\end{array}$ & 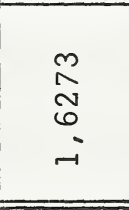 & 1 & $\begin{array}{l}\qquad \\
\infty \\
0 \\
0 \\
-1\end{array}$ & $\begin{array}{l}0 \\
\infty \\
0 \\
10 \\
\end{array}$ & 1 \\
\hline \multirow{3}{*}{ 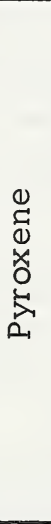 } & 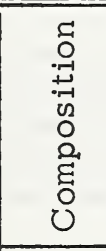 & 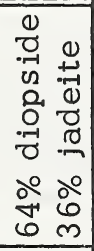 & 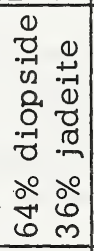 & 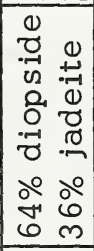 & 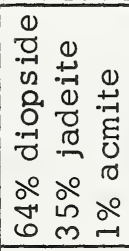 & 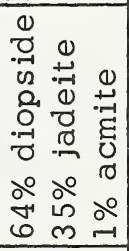 & 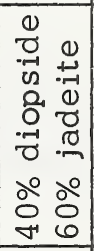 & 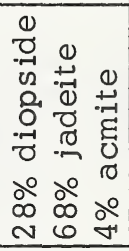 & 1 \\
\hline & 学 & ర & ${ }^{0}$ & ० & ${ }_{m}$ & ${ }_{0}^{0}$ & ${ }_{0}^{0}$ & ${ }_{n}$ & 1 \\
\hline & 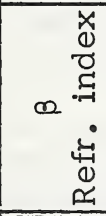 & $\begin{array}{l}0 \\
9 \\
\infty \\
0 \\
-1\end{array}$ & $\begin{array}{l}0 \\
\text { o } \\
\infty \\
0 \\
-1\end{array}$ & $\begin{array}{l}0 \\
\text { क } \\
\infty \\
0 \\
-1\end{array}$ & $\begin{array}{l}\text { W } \\
\infty \\
0 \\
0 \\
-1\end{array}$ & $\begin{array}{l}0 \\
\infty \\
\infty \\
0 \\
-1\end{array}$ & 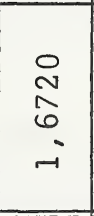 & \begin{tabular}{l}
$\circ$ \\
\multirow{2}{0}{} \\
$-i$
\end{tabular} & 1 \\
\hline \multirow{2}{*}{ 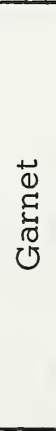 } & 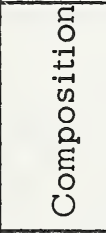 & 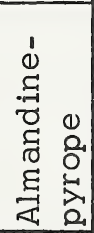 & 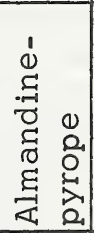 & 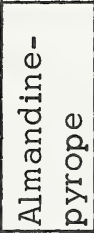 & 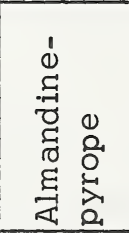 & 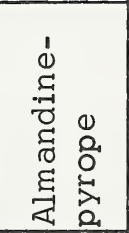 & $\begin{array}{l}0 \\
0 \\
0 \\
0_{1} \\
\lambda_{1}\end{array}$ & $\begin{array}{l}0 \\
0 \\
0 \\
0 \\
D_{1} \\
.1\end{array}$ & 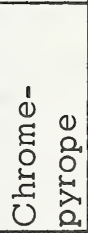 \\
\hline & 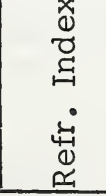 & 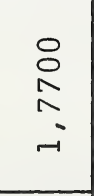 & $\begin{array}{l}0 \\
\hat{0} \\
\\
-i\end{array}$ & 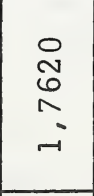 & $\begin{array}{l}0 \\
0 \\
0 \\
-1\end{array}$ & $\begin{array}{l}\circ \\
\circ \\
\vdots \\
-1\end{array}$ & $\begin{array}{l}\stackrel{0}{6} \\
\stackrel{10}{n} \\
i \\
-1\end{array}$ & 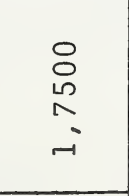 & 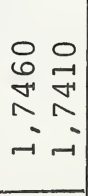 \\
\hline \multicolumn{2}{|r|}{ 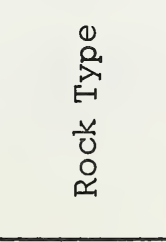 } & 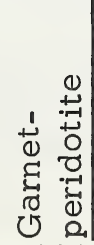 & 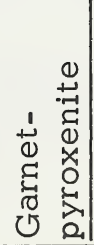 & 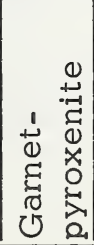 & 湇 & 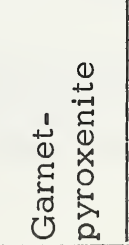 & 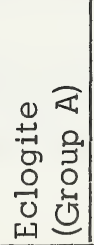 & 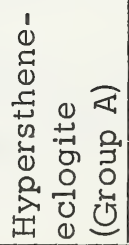 & 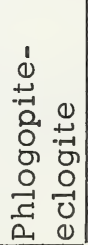 \\
\hline
\end{tabular}

